\title{
Sclerosing Ovarian Tumor with Disrupted Morphology: Significance of Ancillary Tests in Resolving Diagnostic Dilemma
}

\begin{abstract}
Sclerosing stromal tumor is an uncommon subtype of sex cord-stromal tumor of the ovary. It occurs in young females who present with pelvic pain and menstrual irregularities. We present a case of an 18-year-old female who was brought to emergency room with a history of blunt trauma abdomen after road traffic accident. A diagnosis of hemoperitoneum was made, and the patient underwent emergency exploratory laparotomy. An incidental right ovarian mass was found which was removed and sent for histopathological examination. Histomorphology of this lesion showed variable findings with extensive edema and paucicellularity. Few thin wall blood vessels were also observed. The possible differential diagnosis on gross and microscopic examination included sclerosing stromal tumor, fibroma, thecomas, granulosa cell tumor, and edema of the ovary. In view of diagnostic dilemma, special stains for collagen and reticulin were done. In addition, immunohistochemistry (IHC) for CD99, smooth muscle actin, and desmin was put up. A diagnosis of sclerosing tumor of the ovary was made based on histomorphology and findings of ancillary tests. In general, ancillary tests are not required to diagnose sclerosing ovarian tumor as it has characteristic histological features. This case highlights the usefulness of special stains and IHC in sex cord tumors with overlapping microscopic features.
\end{abstract}

Keywords: Atypical, histomorphology, immunohistochemistry, reticulin stain, sclerosing stromal tumor

\section{Introduction}

Sclerosing stromal tumor is a rare benign ovarian neoplasm occurring in young women and accounting for $<5 \%$ of ovarian sex cord-stromal tumors. A pathologist can make a confident correct diagnosis as this tumor bears distinctive histomorphological features that differentiate it from other possible diagnosis. We document a case of sclerosing stromal tumor presenting in an 18-year-old female, posing diagnostic difficulty.

\section{Case Report}

An 18-year-old female was brought to emergency room with complaints of sudden abdominal pain subsequent to blunt trauma abdomen after a road traffic accident (was hit by a car while crossing the road). There was no history of associated vomiting, loss of consciousness, or ear/nose bleed. At presentation, the patient was conscious and oriented. On general examination, pallor was noted. Vital assessed showed a pulse rate of $102 / \mathrm{min}$, blood pressure

This is an open access journal, and articles are distributed under the terms of the Creative Commons Attribution-Non Commercial-ShareAlike 4.0 License, which allows others to remix, tweak, and build upon the work non-commercially, as long as appropriate credit is given and the new creations are licensed under the identical terms.

For reprints contact: reprints@medknow.com of $100 / 50 \mathrm{mmHg}$, and respiratory rate of 18/min. Per abdomen examination revealed abdominal distension and guarding with diffuse rebound tenderness. Bowel sounds were absent. No abnormality was detected on per rectal examination done. There were superficial abrasions present on the left hand and left knee. Her menstrual history was 4/30-day regular cycles with normal flow. Her bowel and bladder habits were normal. There was no significant past history, and the patient was totally asymptomatic before this episode of acute abdominal pain. In view of hemodynamic instability and for evaluation of blood in peritoneum, rapid bedside ultrasound examination was done which reflected positive test on focused assessment with sonography for trauma (FAST). A clinical diagnosis of blunt trauma abdomen with hemoperitoneum was made, and the patient was taken for emergency exploratory laparotomy.

Per-operative findings

A large right ovarian mass was noted. Diffuse ooze was noted from raw peritoneal

\footnotetext{
How to cite this article: Rao S, Muralitharan S, Narayanan CD. Sclerosing ovarian tumor with disrupted morphology: Significance of ancillary tests in resolving diagnostic dilemma. Indian J Med Paediatr Oncol 2018;39:408-12.
}

\section{Shalinee Rao, Susruthan \\ Muralitharan', Cunnigaiper Dhanasekharan Narayanan ${ }^{2}$}

Department of Pathology and Laboratory Medicine, All India Institute of Medical Sciences, Rishikesh, Uttarakhand, Departments of ${ }^{1}$ Pathology and ${ }^{2}$ Surgery, Sri Ramachandra Medical College and Research Institute, Chennai, Tamil Nadu, India

Address for correspondence Dr. Shalinee Rao, Department of Pathology and Laboratory Medicine, All India Institute of Medical Sciences, Rishikesh - 249 203, Uttarakhand, India.

E-mail: shalineerao@gmail.com

Access this article online Website: www.ijmpo.org DOI: 10.4103/ijmpo.ijmpo_21_17 Quick Response Code:

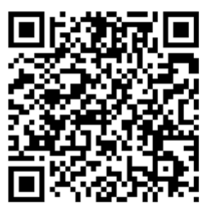


surface. Right oophorectomy was performed and sent for routine histopathological examination. Due to an elevated international normalized ratio (INR), hemostatic pads in the abdomen were placed and abdomen was closed over a drain in the pelvis. The patient was intubated and shifted to Intensive Care Unit. After $48 \mathrm{~h}$, INR normalized and the patient was taken up for laparoscopic retrieval of hemostatic packs.

Ovarian mass received grossly measured $7.5 \mathrm{~cm} \times 5 \mathrm{~cm} \times 3.5 \mathrm{~cm}$. External surface was nodular. Cut section appeared predominantly solid with few tiny cystic changes. Solid areas appeared soft to firm, homogeneous, gray white with yellow areas and at areas appeared edematous. Omentum sent separately measured $2 \mathrm{~cm} \times 1 \mathrm{~cm} \times 0.3 \mathrm{~cm}$. On microscopy, ovarian lesion was composed of aggregates of the spindle to oval cells having vesicular nucleus with intervening zones showing aggregates of plump oval cells resembling luteinized cells [Figures 1 and 2]. Large areas showed edematous change and thin-walled vessels showing irregular branching [Figure 3]. Focal areas showed hyalinization and sclerosis. No mitotic figures were seen in multiple sections studied. Histomorphological features were indicative of sex cord-stromal tumor and edema of the ovary with possibilities suggestive of juvenile granulosa cell tumor, fibrothecoma, and sclerosing ovarian tumor. Immunohistochemistry (IHC) workup and special stains were put up for further diagnosis. Omentum showed only fibrofatty tissue.

Special stain Masson's trichrome showed focal positivity for collagen [Figure 4b]. Reticulin stain showed a mild increase in reticulin fibers around blood vessels [Figure 4a]. Imunohistochemical stains showed CD99 negativity. Focal positivity for smooth muscle actin was noted in blood vessels and stroma [Figure 4c], and scattered focal positivity was noted with desmin immunostain [Figure 4d]. In view of histopathological changes, histochemical and

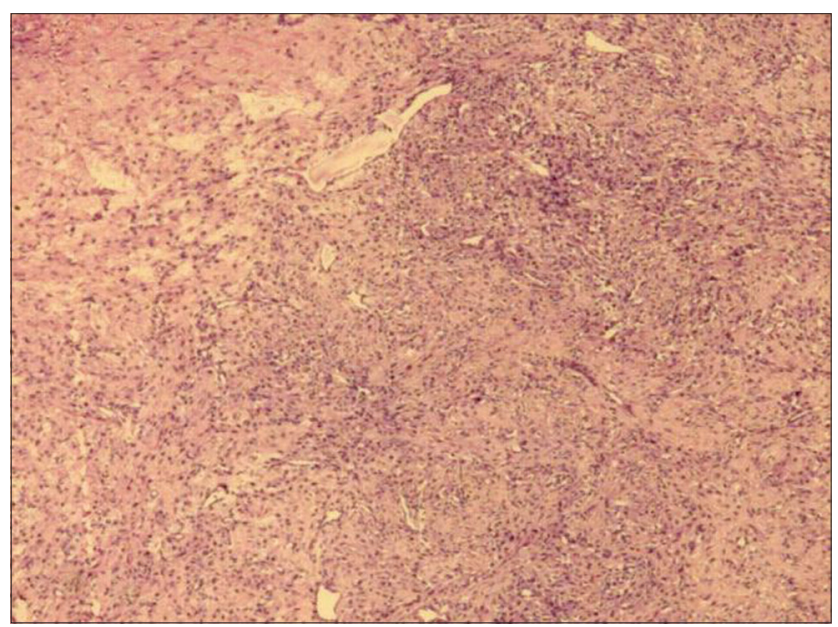

Figure 1: Section showing cells in diffuse pattern and as aggregates in abundant stroma (H and $\mathrm{E} \times 20$ )
IHC findings, a final diagnosis of sclerosing stromal tumor of the ovary was rendered.

\section{Discussion}

Essential parameters required for accurately diagnosing ovarian tumors include clinical information such as patient age, presenting sign, and symptoms and pathological examination findings such as gross and microscopic features. Germ cell tumors of the ovary may show elevated tumor markers as well. Sex cord-stromal tumors may show clinical manifestations due to hormonal alterations (estrogenic or androgenic). In reference to the present case, in which a young female patient of 18 years of age who had been asymptomatic showed an incidental right ovarian mass, the most probable diagnosis on initial routine histopathological examination favored sex cord-stromal tumor and edema of the ovary. Sex cord-stromal tumor of the ovary is subcategory of neoplasms that comprises granulosa cells, theca cells, Sertoli cells, Leydig cells, and fibroblasts of stromal origin, singly or in various proportions. $^{[1]}$ Sex cord-stromal tumors comprise about $8 \%$ of all ovarian tumors. ${ }^{[2]}$ In the present case, the difficulty to make a probable diagnosis even after routine pathological examination was encountered due to distorted tumor morphology.

Sclerosing stromal tumor is very uncommon benign ovarian tumor accounting for $2 \%-6 \%$ of all ovarian sex cord-stromal tumors. ${ }^{[3]}$ It occurs in younger women, usually in the second and third decades, and is predominantly a hormonally inactive sex cord-stromal ovarian tumor. The presenting symptoms include disturbance of menstrual cycles, pelvic pain abdominal discomfort, and rarely hormonal manifestation such as hirsutism. The tumor is usually unilateral and can be rarely bilateral. Grossly, the tumor is well-circumscribed solid gray white having occasional yellowish foci with edematous and cystic areas. In the present case, on gross inspection, the tumor was

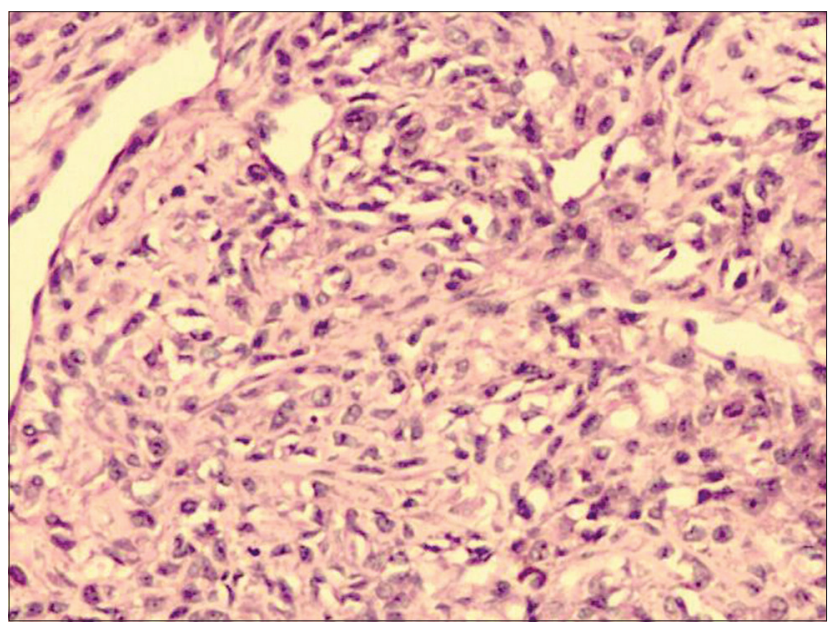

Figure 2: Cellular areas composed of oval-to-spindle cells with plump vesicular nuclei and pale eosinophilic to clear cytoplasm $(H$ and $E \times 200)$ 


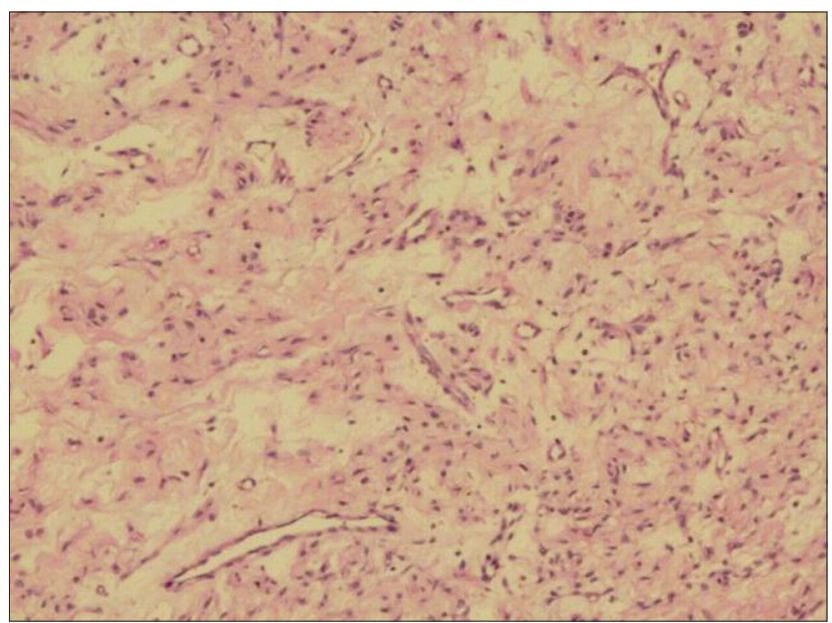

Figure 3: Large areas of edematous change and many thin-walled branching vascular channels noted in stroma ( $\mathrm{H}$ and $\mathrm{E} \times 40)$

distorted and edematous. On microscopy, it displays a distinctive appearance with a pseudolobular architectural pattern having alternate areas of cellular zones and paucicellular fibrous zones with fibroblasts and rounded vacuolated cells. ${ }^{[4]}$ Scattered throughout within the tumor are thin-walled dilated arborizing vascular channels having a "hemangiopericytoma-like" appearance. The neoplastic cells adjoining the blood vessels are often plump to polygonal, vacuolated (due to the presence of lipid), or fairly myoid in appearance. Rarely, these cells may exhibit signet-ring features in occasional cases. It is possibly derived from the perifollicular myoid stromal cells. ${ }^{[5]}$ In the present case study, there was a loss of lobular pattern with large edematous areas.

Fibromas and thecomas are benign stromal tumors of the ovary and can occur at any age but are seen more frequently in middle age, over 40 years of age. Luteinized variants of thecoma tend to occur at younger age. ${ }^{[6]}$ Fibroma is the most common subtype of the sex cord-stromal tumors, accounting for about two-thirds of neoplasms. Ascites is the most common abdominal symptom being present in over $10 \%$ of cases. ${ }^{[7]}$ In $10 \%$ of cases with bilateral lesions, it appears to be associated with basal-cell nevus syndrome . ${ }^{[8]}$ Grossly, fibroma is a diffuse solid tumor varying in size from small to large lesions. Larger tumors have a smooth or slightly bosselated external surface and vary in consistency from edematous or rubbery to hard. Cut surface is whitish, faintly whorled and can show areas of cystic degeneration. Microscopically, crisscrossing bundles of plump spindle cells are seen laying down abundant collagen. Some of the tumor may also show variable degree of intercellular edema. Neoplastic cells of fibromas may contain intracytoplasmic lipid. Uncommonly, fibromas can feature varied cellularity and prominent vascularity, which may result in a misdiagnosis of sclerosing stromal tumor. ${ }^{[8]}$ Thecoma is also benign spindle-cell neoplasm and differs from fibroma as it is often hormonally functioning, secreting estrogen. Usually, it is a unilateral tumor and

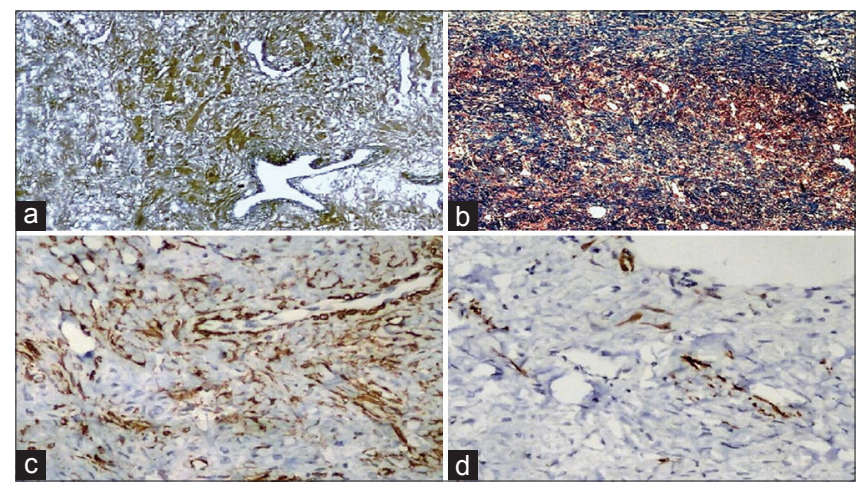

Figure 4: (a) Mild increase in reticulin fibers only around blood vessels (Reticulin stain $\times 40$ ); (b) Bluish stained collagen deposition focally present (Masson trichrome $\times 20$ ); (c) Focal immunopositivity noted in blood vessel and stroma (Sma immunostain $\times 100$ ); (d) Scant focal immunopositivity noted within stroma (Desmin immunostain $\times 100$ )

ranges in size from small to large solid masses. Sectioning reveal solid, firm to rubbery consistency with yellowish areas. Histopathological examination shows nodules composed of cells resembling the ovarian theca interna cells appearing oval to round, with abundant pale vacuolated cytoplasm filled with lipid (due to estrogen secretion) with intervening areas comprising of bands of fibrous connective tissue and hyalinized areas. Edema or myxoid change may be a conspicuous feature. Some tumors show an occasional focus of dystrophic calcification. Rarely, tiny nests of granulosa cells may be seen and are termed as "thecomas with minor sex cord elements." Luteinized thecoma is a subtype of thecoma and is associated with sclerosing peritonitis. Microscopically, it is characterized by alternating hypercellular and hypocellular areas, edema and microcystic areas. ${ }^{\left[{ }^{9]}\right.}$ Few mitotic figures may be evident.

Juvenile granulosa cell tumor is sex cord-stromal tumor presenting in the first three decades of life. Patients in prepubertal age group present with isosexual pseudoprecocity while in postpubertal age group, presentation includes abdominal pain or with menstrual disturbance or amenorrhea. ${ }^{[10]}$ Juvenile granulosa cell tumor is bilateral in $2 \%$ of cases and can vary in size from small lesions to large tumor mass. It can rupture in $10 \%$ of cases. ${ }^{[10]}$ Grossly, it is a solid cystic neoplasm with clear or hemorrhagic fluid. Solid areas may be hard and rubbery or soft in consistency. Cut section showed yellow-tan areas with necrosis and hemorrhage. Histopathology displays solid cellular areas with focal follicle formation. Neoplastic cells are arranged in diffuse pattern and as nodules separated by fibrous septa. The follicles are of smaller sizes and their lumens filled with basophilic or eosinophilic fluid. Follicles are lined by granulosa cells which appear round to polygonal, spindle shaped with scant amphophilic cytoplasm and having indistinct cytoplasmic borders with round, oval, or angular nuclei. Diagnostic problems arise when tumor cells in solid areas are arranged in diffuse pattern or due to the presence of fibrous stroma with focal luteinization and/or edema. 
Massive ovarian edema is an unusual cause of unilateral ovarian mass in women of a reproductive age group and can be mistaken for neoplasm. Clinical features include abdominal pain, abdominal mass, menstrual disturbance, virilization, precocious puberty, and Meigs syndrome (with ascites and pleural effusion). This condition occurs due to partial torsion of the mesovarium leading to interference of venous/lymphatic drainage.

Grossly, there is a marked enlargement of the ovary with soft, gelatinous watery cut surface. Sectioning of this lesion exudes out watery fluid. Microscopically, it is hypocellular lesion with extensive edematous stroma compressing the cortical stroma. Collection of luteinized stromal cells may also be seen.

For a pathologist, a diagnosis of sclerosing ovarian tumor is usually straightforward and relies on its characteristic gross and unique histologic features including pseudolobulation, sclerosis, and prominent vascularity. Hematoxylin and eosin (H and $\mathrm{E})$ stained sections provide adequate information so that ancillary tests such as histochemistry and IHC may not be required and hence avoid extra expenditure. However, in the present case due to loss of pseudolobular architecture, intense edema, and overlapping morphological features, a clear-cut diagnosis was difficult. It necessitated a further workup to resolve the diagnostic dilemma. Since it was essential, histochemical stains and panel of IHC stains were put up for further evaluation and diagnosis. In granulosa cell tumor, reticulin fibers are seen surrounding aggregates and nests of tumor cells while in thecoma, fibrils invest around individual cells. In sclerosing ovarian tumor, reticulin fibers are noted mainly around blood vessels as noted in our case. In our case, abundant edema was noted and there was focal presence of collagen, which was highlighted by Masson trichrome stain. In contrast to this, collagen in fibromas is abundant and can be demonstrated by special stains for collagen such as Masson trichrome and Van Gieson stains. Oil Red O or Sudan black on fresh tissue can be utilized to confirm intracytoplasmic fat stains in lipidized cells of thecoma and luteinized cells.

Inhibin and calretinin are the usual immunomarkers positive in sex cord-stromal tumor of the ovary, and expression of these is stronger and more diffuse in granulosa cell tumors and Sertoli and Sertoli Leydig cell tumors than in fibroma or thecoma. Vimentin, smooth muscle actin, and desmin are other immunomarkers which show cytoplasmic positivity in sclerosing stromal tumors. The pattern of immunopositivity is also important. In sclerosing stromal tumor, desmin and smooth muscle actin delineate arborizing vessels as well focal positivity is noted in stroma. ${ }^{[4]}$ The less reliable immunomarkers positive in sclerosing stromal tumors are WT1 and CD99. In our case, CD99 was negative while desmin and smooth muscle actin showed focal positivity in stroma and blood vessel wall. Estrogen receptor and progesterone receptor may be expressed in many granulosa cell tumors. Thecoma shows positivity for inhibin and calretinin and a stronger expression for inhibin favors thecoma over fibroma. In general, for sclerosing stromal tumor and fibromas, routine $\mathrm{H}$ and $\mathrm{E}$-stained sections are enough to correctly diagnose as both these tumors have distinctive features and IHC may not be required. It is only when histomorphology has overlapping features with mixed pattern creating confusion to the pathologist that IHC would be used to confirm a diagnosis and rule out other differential diagnosis.

Sclerosing stromal tumor of the ovary can be treated effectively by enucleation or unilateral ovariotomy. ${ }^{[11]}$

\section{Conclusion}

Although a diagnosis of sclerosing stromal tumor of the ovary can be made on routine histopathological sections, in situations with morphological ambiguity, special stains such as reticulin and immunohistochemical stains such as smooth muscle actin and desmin can contribute immensely in identifying this rare benign tumor.

\section{Declaration of patient consent}

The authors certify that they have obtained all appropriate patient consent forms. In the form the patient(s) has/have given his/her/their consent for his/her/their images and other clinical information to be reported in the journal. The patients understand that their names and initials will not be published and due efforts will be made to conceal their identity, but anonymity cannot be guaranteed.

\section{Financial support and sponsorship}

Nil.

\section{Conflicts of interest}

There are no conflicts of interest.

\section{References}

1. Mircea R, Anton E, Anton C, Tărniceriu C, Nedelcu AH, Pricop FZ, et al. Specific features of the stromal ovarian tumors. Rev Med Chir Soc Med Nat Iasi 2012;116:1123-30.

2. Atram M, Anshu, Sharma S, Gangane N. Sclerosing stromal tumor of the ovary. Obstet Gynecol Sci 2014;57:405-8.

3. Young RH. Sex cord-stromal tumors of the ovary and testis: Their similarities and differences with consideration of selected problems. Mod Pathol 2005;18 Suppl 2:S81-98.

4. Peng HH, Chang TC, Hsueh S. Sclerosing stromal tumor of ovary. Chang Gung Med J 2003;26:444-8.

5. Lam RM, Geittmann P. Sclerosing stromal tumor of the ovary. A light, electron microscopic and enzyme histochemical study. Int J Gynecol Pathol 1988;7:280-90.

6. Renaud MC, Plante M, Roy M. Ovarian thecoma associated with a large quantity of ascites and elevated serum CA 125 and CA 15-3. J Obstet Gynaecol Can 2002;24:963-5.

7. Sivanesaratnam V, Dutta R, Jayalakshmi P. Ovarian fibroma - Clinical and histopathological characteristics. Int $\mathrm{J}$ Gynaecol Obstet 1990;33:243-7.

8. Puri WS, Shobha M. Bilateral ovarian fibromas in a young 
patient: A rare occurrence. Chron Young Sci 2014;5:69-71.

9. Clement PB, Young RH, Hanna W, Scully RE. Sclerosing peritonitis associated with luteinized thecomas of the ovary. A clinicopathological analysis of six cases. Am J Surg Pathol 1994;18:1-3.

10. Outwater EK, Wagner BJ, Mannion C, McLarney JK, Kim B.
Sex cord-stromal and steroid cell tumors of the ovary. Radiographics 1998;18:1523-46.

11. Gwin K, Mariño-Enríquez A, Martel M, Reyes-Múgica M. Sclerosing stromal tumor: An important differential diagnosis of ovarian neoplasms in childhood and adolescence. Pediatr Dev Pathol 2009;12:366-70. 